\section{$\underset{\substack{\text { hommes } \\ \text { \& migrations }}}{ }$}

\section{Hommes \& migrations}

Revue française de référence sur les dynamiques

migratoires

1307 | 2014

L'Afrique qualifiée dans la mondialisation

\title{
La diaspora kurde en Suède
}

\section{Barzoo Eliassi}

\section{(2) OpenEdition \\ Journals}

\section{Édition électronique}

URL : http://journals.openedition.org/hommesmigrations/2902

DOI : 10.4000/hommesmigrations.2902

ISSN : 2262-3353

\section{Éditeur}

Musée national de l'histoire de l'immigration

\section{Édition imprimée}

Date de publication : 1 juillet 2014

Pagination : 144-149

ISBN : 978-2-919040-28-5

ISSN : $1142-852 X$

\section{Référence électronique}

Barzoo Eliassi, «La diaspora kurde en Suède », Hommes \& migrations [En ligne], 1307 | 2014, mis en ligne le 15 janvier 2015, consulté le 22 septembre 2020. URL : http://journals.openedition.org/ hommesmigrations/2902 ; DOI : https://doi.org/10.4000/hommesmigrations.2902 


\section{REPÉRAGE}

\section{LA DIASPORA KURDE EN SUĖDE}

BARZOO ELIASSI, chercheur en sciences sociales et politiques à l'Institut des migrations internationales, université d'Oxford, et au Centre d'études sur le Moyen-Orient, université de Lund en Suède.

'émergence de la diaspora kurde en Suède va Lde pair avec la revendication de son identité et son adhésion à un discours "kurdiste". Ce dernier marque la transition entre une position de minorité et une identité nationale pan-kurde visant à renommer le pays d'origine "Kurdistan" et à le réhabiliter en tant que site principal de l'identité kurde. II vise également à replacer la notion de "kurdicité" au sein de frontières géographiques définies au Moyen-Orient et d'associer l'identité kurde à un territoire particulier.

Nous explorerons les différentes manières dont les Kurdes conjuguent le maintien de leur identité avec leur vie quotidienne au sein de la société suédoise. L'expérience diasporique des Kurdes en Suède oscille entre phénomènes d'inclusion et d'exclusion, droits de citoyenneté et discriminations, appartenance et non-appartenance. L'article s'appuie sur un travail de terrain extensif mené auprès de migrants kurdes en Suède, qui donna lieu au premier livre publié sur cette diaspora', mais également sur un projet de recherche en cours à l'université d'Oxford, centré sur des expériences individuelles et collectives autour de l'apatridie parmi des Kurdes au sein de l'Union européenne. Nombre d'immigrés kurdes, intellectuels, artistes, écrivains et activistes politiques sont arrivés en Suède suite aux conflits ethnopolitiques entre les Kurdes et les États d'Iran, d'Irak, de Syrie et de Turquie. Le contexte historique de cette immigration explique la nature fortement politisée de nombreux Kurdes en Suède. L'élite kurde a joué un rôle décisif dans la consolidation d'une identité nationale kurde et a milité pour sa reconnaissance au travers d'activités mobilisatrices au sein d'associations, via des diffusions satellitaires et radio et grâce à Internet. Résultat direct des activités politiques des migrants kurdes en Occident, la question kurde n'est plus intrinsèque aux pays du Moyen-Orient dans lesquels ils habitent. D'ordre tout d'abord régional, puis européen, cette question revêt désormais une dimension internationale.

Paradoxalement, l'oppression d'État envers les Kurdes au Moyen-Orient a favorisé la naissance d'un réseau d'activisme politique kurde transnational ayant pour but de lutter contre les politiques de répression de manière plus tangible que par des actions de guérilla.

Le facteur d'émigration a également fourni aux Kurdes de tout le Kurdistan un espace politique démocratique en Occident, dans lequel négocier, dépasser et intégrer bon nombre de leurs différences. 


\section{L'accueil favorable de la diaspora kurde en Suède}

La Suède compte quelque 70000 Kurdes en provenance de différentes régions du Kurdistan au Moyen-Orient. Elle a longtemps été dépeinte comme un modèle de terre d'accueil : État-providence tolérant, égalitaire, multiculturel, appliquant à tous, y compris aux communautés immigrées, les mêmes droits de citoyenneté et du travail, ainsi que les mêmes droits sociaux. De plus, l'État-providence suédois a permis aux populations immigrées d'accéder à une citoyenneté civile, politique et sociale, selon une logique de justice distributive. Les conditions d'accès à la citoyenneté suédoise sont parmi les plus souples d'Europe occidentale. Aussi, depuis 1975, les personnes n'ayant pas obtenu la nationalité suédoise ont-elles le droit de voter aux élections locales et régionales. Des associations kurdes ont bénéficié d'aides financières de l'État et représentent pour la communauté des espaces d'échanges autour d'intérêts collectifs. Les étudiants sont autorisés à suivre des cours dans leur langue maternelle jusqu'à l'école secondaire supérieure. Alors que la pratique de la langue kurde était interdite en Turquie durant des décennies, le modèle multiculturel suédois, favorable et ouvert, ainsi que le dynamisme de la diaspora ont permis le renouveau du kurmandji, variante de la langue kurde. C'est en Suède que les activités culturelles kurdes sont les plus nombreuses derrière le Kurdistan irakien et les Républiques du Caucase. Aussi, la diaspora kurde en Suède abrite-t-elle la plus grande production romanesque et littéraire kurde. On parle d'ailleurs d'"école suédoise de littérature kurde ${ }^{2}$ " pour définir l'influence de la diaspora en Suède sur la production littéraire kurde.
Pour résumer, d'un point de vue international, le multiculturalisme et l'État-providence suédois ont servi de modèle à de nombreux États. Les Kurdes ont énormément bénéficié de la liberté politique et des principes démocratiques suédois. La Suède a servi de vecteur à un Kurdistan élargi en matière d'activités culturelles et linguistiques et de droits que les mouvements kurdes peinent à obtenir au Moyen-Orient. Pour preuve, la Suède abrite l'une des plus grandes bibliothèques kurdes au monde et compte 7 parlementaires d'origine kurde qui soutiennent les droits des Kurdes en Iran, Irak, Syrie et Turquie. Ces parlementaires sont dispersés dans différents partis politiques (droite, libéral, gauche, écologique), même si, traditionnellement, ils étaient issus de partis de gauche. Le réseau parlementaire suédois pour le Kurdistan fut créé en 2006 dans le but de resserrer les liens entre, d'un côté, les organisations démocratiques et les politiciens suédois, et, de l'autre, l'électorat issu de l'immigration kurde et le Kurdistan. Bien que relativement récente, la diaspora kurde en Suède compte parmi l'une des mieux organisées, elle est également une médiatrice efficace de la situation politique des Kurdes au Moyen-Orient auprès du peuple suédois. Les questions autour des politiques identitaires kurdes ne se sont pas taries avec la migration et l'exil, bien au contraire. Elles se sont intensifiées grâce au climat de liberté politique et aux possibilités ouvertes en Europe occidentale.

\section{La sensibilisation des principaux partis politiques suédois à la cause kurde}

Le 16 mars 2008, quelque 500 personnes allumaient des milliers de bougies à Stockholm en hommage aux victimes de Halabja (ville kurde 


\section{REPÉRAGE}

située au Kurdistan irakien), qui périrent dans des attaques à l'arme chimique lancées par le régime de Saddam Hussein le 16 mars 1988. Parmi les personnes présentes à la cérémonie, de nombreux hommes politiques suédois connus de la sphère publique tels que Mona Sahlin, ancien chef socialdémocrate, Lars Ohly, ancien chef du parti de gauche, et Sven-Otto Littorin, ancien ministre du Marché du travail du parti au pouvoir Moderaterna.

Ce dernier souligna que : "Les Kurdes représentent un atout pour la Suède et un bon exemple d'intégration réussie." Sur le même ton, Mona Sahlin, tout en déplorant les attaques chimiques sur la ville de Halabja, insista sur la nécessité de participer à une telle cérémonie car les "Kurdes occupent une place importante en Suède".

L'intégration réussie des Kurdes dans la société suédoise ne rime donc pas nécessairement avec perte d'identité. Au contraire, cette position peut servir au lobbying kurde et à la mobilisation d'acteurs potentiels pro-kurdes dans l'ensemble de la société, pour influencer la politique étrangère suédoise vis-à-vis des Kurdes et les politiques des pays concernés au Moyen-Orient.

Le poids de la diaspora kurde s'est vérifié en 2012, lorsque le Parlement suédois, tous partis politiques confondus, a unanimement qualifié de génocide les atrocités commises par le régime de Saddam Hussein contre les Kurdes.

Si les membres de la diaspora s'investissent dans la défense des droits kurdes, ils militent également contre les structures anti-démocratiques de certains partis politiques kurdes et pour l'égalité des sexes, que ce soit dans le pays d'origine ou au sein de la diaspora.

\section{Au-delà d'une identité plurielle}

La diaspora kurde a donc fortement marqué la société d'accueil. Mieux, elle y occupe une place de choix dans les différents débats sociétaux. Des auteurs et débatteurs kurdes comme Lawen Mohtadi, Dilsa Demirberg, Mustafa Can et des humoristes comme Soran Ismail et Özz Nûjen s'expriment régulièrement sur des questions sociales et politiques. Ces actions publiques témoignent de la volonté de créer un nouvel imaginaire national en Suède, qui dépasse l'essentialisme ethnique et véhicule l'idée d'une certaine "suéditude" comprenant l'apparence, la culture, l'histoire, la religion, les relations hommes-femmes, etc. Ces personnalités, dont la parole est publique, prennent part aux discours sur l'identité suédoise et sur les expériences migratoires. Néanmoins, cette participation ne les rend pas indifférents à la situation politique des Kurdes.

Pour les membres de la diaspora kurde, la vie en Suède revêt deux facettes : la culture politique démocratique, d'une part, et, de l'autre, les inégalités ethniques et structurelles qui entravent au quotidien leurs droits de citoyen. Les migrants kurdes font clairement la différence entre la notion officielle de citoyenneté suédoise et la réalité. L'identité d'immigré est une identité à la fois imposée et vécue, car leur altérité et leurs différences culturelles sont régulièrement critiquées comme indésirables. Beaucoup sont victimes d'abus racistes et de discriminations à l'école, au travail, dans le logement, les démarches légales, etc. Cette discrimination renforce leur sentiment d'identité kurde et aiguise leur conscience de ne pas être acceptés pleinement par la société suédoise.

Le sport est un autre lieu d'expression de réussite pour les Kurdes. Le Dalkurd FF est un club 
de football fondé en 2004 qui joue dans la troisième meilleure division du pays. Malgré son jeune âge, il est le club le plus suivi de Suède sur Facebook avec plus de 223459 "J'aime" (à la date du 3 avril 2014). Le nom "Dalkurd" évoque la connexion de l'identité kurde avec Dalarna, une région en Suède. II dénote une identité transnationale liant la Suède au Kurdistan, mais également une régionalisation de l'identité kurde en Suède. D'autres clubs, tels Uppsalakurd et Skanekurd, ont été créés à la suite du Dalkurd. Le football est un sport international et un moyen pour les Kurdes d'obtenir une visibilité et une reconnaissance aux yeux du monde, mais aussi de véhiculer leur identité réprimée par les nationalismes dominants d'Iran, d'Irak, de Syrie et de Turquie.

\section{De "kurde" à "kurdiste" : la création d'une identité "kurdiste" en ligne}

En janvier 2014, deux jeunes Suédo-Kurdes créaient une page Facebook intitulée "Ez kurdistanî me/ Min kurdistanî m" ("Je suis "kurdiste"'). Sur cette page, des Kurdes du monde entier postent leur photo et l'accompagnent du message qu'ils sont "kurdistes". Des artistes, poètes, figures publiques et hommes politiques ont participé à cette campagne aux côtés de Kurdes lambda. En seulement trois mois, la page Facebook a récolté 20986 "J'aime". Lorsque j'ai demandé aux deux jeunes initiateurs de la campagne les raisons de ce projet, l'un d'eux a déclaré : "Nous ne devrions plus nous définir comme Irakiens, Turcs, Iraniens, Syriens ou même Kurdes irakiens, Kurdes iraniens, Kurdes turcs ou Kurdes syriens. Nous devrions rejeter ces définitions des États occupants et plutôt nous revendiquer d'une identité "kurdiste" avec pour pays le
Kurdistan, seul unificateur de tous les Kurdes. Cela nous aiderait à nous identifier les uns aux autres et à nous sentir plus proches. Prenez l'exemple des Palestiniens, ils ne se définissent jamais comme étant israéliens, ils se disent toujours Palestiniens et veulent le rester. Mais nous les Kurdes, nous acceptons la dénomination adoptée par les États ennemis et nous en oublions notre identité kurde." Par opposition à une identité kurde qui n'est pas, par définition, rattachée à une géographie ou à un territoire particulier, l'identité "kurdiste", elle, englobe un imaginaire national s'inscrivant au sein de frontières géographiques bien définies au Moyen-Orient. La géographie est donc au centre de la création d'une identité natio-
Équipe de footbal Dalkurd (c) JonEs KızIL

nale, souvent ancrée dans un territoire. Pour les Kurdes, cette identité territoriale a longtemps fait partie de leur lutte pour la reconnaissance, l'autonomie et l'indépendance. Ils comprennent que dans un monde d'États-nations, il est nécessaire de pouvoir localiser son identité sur la carte 


\section{REPÉRAGE}

mondiale et de projeter une image explicitement définie à l'extérieur. La campagne "Je suis "kurdiste"' montre clairement que l'appellation joue un rôle important dans la construction d'un nouvel imaginaire national, mais aussi dans la réappropriation de territoires turquifiés, arabisés ou persanisés, et où la présence kurde a été marginalisée ou effacée. Cette campagne tente donc de subvertir les tracés géographiques et politiques actuels qui ont éclaté les Kurdes en quatre groupes nationaux et quatre États. La force de la diaspora réside dans son habileté à initier un courant capable de transformer les identités politiques (d'“Irakien" à "Kurdiste", par exemple) et de créer de nouvelles formes de solidarité entre les Kurdes à identités fragmentées par la création de l'Iran, I'Irak, la Turquie et la Syrie. L'absence d'un État kurde doté d'une administration politique unifiée a aussi contribué à la division entre Kurdes. Malgré cela et grâce aux nouvelles technologies d'information, les Kurdes se regroupent, se rapprochent et échangent sur leur identité et leur diversité par le biais de réseaux sociaux tels que Facebook.

\section{Les symboliques \\ de la région du Kurdistan pour la diaspora kurde}

Une des préoccupations récurrentes au sein des diasporas est celle de la terre d'origine et du retour. lémergence d'un État "quasi kurde" dans la région du Kurdistan irakien a fourni aux exilés kurdes un espace de vie sûr, où ils peuvent pleinement vivre leur "kurdicité" sans risques d'assimilation et d'oppression ethnique. La construction d'aéroports à Erbil et à Souleimania a relié le Kurdistan à sa diaspora. Des Kurdes de tout le Kurdistan visitent cette région qualifiée d'expérience historique sans précédent, mais aussi lieu de répit de la domination dont les Kurdes ont fait l'objet en Irak.

Des membres de la diaspora kurde évoquent les conditions de retour avant la construction de ces aéroports. Ils se souviennent encore de l'expérience douloureuse et de l'attitude humiliante des gardes-frontières turcs à Ibrahim Khalil, point qui sépare la partie turque du Kurdistan de la région du Kurdistan en Irak. Le contexte politique de l'époque était marqué par le refus de leur reconnaître une identité kurde en Turquie. Face à ce déni, la région du Kurdistan représentait une entité kurde et la résistance au discours virulent de l'État turc visant à éradiquer toute "kurdicité". L'identité kurde est non seulement chère aux Kurdes d'Irak mais aussi à ceux des autres régions du Kurdistan. Beaucoup ont vu dans la partie libérée du Kurdistan un signe d'espoir pour parvenir à leur tour à un degré d'autonomie et pour s'affranchir ainsi de la logique d'État oppressante dont ils sont victimes. Pourtant, certains migrants kurdes se sont montrés critiques envers la région du Kurdistan en réclamant plus de démocratie, l'égalité hommesfemmes et une société civile plus forte et libre de toute ingérence des partis politiques kurdes dans le quotidien des habitants. Les droits des minorités restent aussi une question importante. Un membre de la diaspora kurde expliquait : "La première chose à faire pour un État kurde est de protéger les minorités car toute la violence que nous avons subie en tant que Kurdes doit nous servir d'exemple pour ne pas appliquer le même sort à d'autres minorités." 


\section{Conclusion}

L'émergence de la diaspora kurde en Suède résulte d'un manque de libertés économiques et politiques au Moyen-Orient. Grâce à la spécificité du système multiculturel de la Suède, les Kurdes ont pu se mobiliser et s'engager dans les politiques de leur pays d'origine sans pour autant s'isoler du reste de la société suédoise. La diaspora kurde a permis de construire un pont entre la Suède et le Kurdistan. Les Kurdes en Suède ne souhaitent pas choisir entre leur double identité mais plutôt faire cohabiter les deux en symbiose. Pour les jeunes, cela est d'autant plus vrai que leur identité ne peut se construire que sous l'angle de la multiplicité. Lémergence de la région du Kurdistan en Irak a permis aux Kurdes de se rapprocher de leur foyer imaginaire : le Kurdistan. Tant que les Kurdes seront victimes de discrimination au Moyen-Orient, la diaspora restera sans doute politisée, revendiquant sa position de victime pour instiguer une mobilisation politique transnationale visant à modifier les structures d'État opprimantes au Moyen-Orient. Les Kurdes de Suède s'inscrivent à la tête d'un tel mouvement. Beaucoup prennent conscience qu'ils ne sont pas arrivés en Suède pour s'assimiler complètement ou faire fi de leur identité kurde, mais plutôt avec l'intention de continuer à se sentir kurdes sans pour autant rester en marge de la société suédoise. 